\title{
A $q$-analogue of Graham, Hoffman and Hosoya's Theorem
}

\author{
Sivaramakrishnan Sivasubramanian \\ Department of Mathematics \\ Indian Institute of Technology, Bombay \\ krishnan@math.iitb.ac.in
}

Submitted: Apr 23, 2009; Accepted: Apr 7, 2010; Published: Apr 19, 2010

Mathematics Subject Classification: 05A30, 05C12

\begin{abstract}
Graham, Hoffman and Hosoya gave a very nice formula about the determinant of the distance matrix $D_{G}$ of a graph $G$ in terms of the distance matrix of its blocks. We generalize this result to a $q$-analogue of $D_{G}$. Our generalization yields results about the equality of the determinant of the mod-2 (and in general mod- $k$ ) distance matrix (i.e. each entry of the distance matrix is taken modulo 2 or $k$ ) of some graphs. The mod- 2 case can be interpreted as a determinant equality result for the adjacency matrix of some graphs.
\end{abstract}

\section{Introduction}

Graham and Pollak (see [3]) considered the distance matrix $D_{T}=\left(d_{u, v}\right)$ of a tree $T=(V, E)$. For $u, v \in V$, its distance $d_{u, v}$ is the length of a shortest (in this case unique) path between $u$ and $v$ in $T$ and since any tree is connected, all entries $d_{u, v}$ are finite. Let $D_{T}$ be the distance matrix of $T$ with $|V|=n$. They showed a surprising result that $\operatorname{det}\left(D_{T}\right)=(-1)^{n-1}(n-1) 2^{n-2}$. Thus, the determinant of $D_{T}$ only depends on $n$, the number of vertices of $T$ and is independent of T's structure.

Graham, Hoffman and Hosoya [2] proved a very attractive theorem about the determinant of the distance matrix $D_{G}$ of a strongly connected digraph $G$ as a function of the distance matrix of its 2-connected blocks (also called blocks). Denote the sum of the cofactors of a matrix $A$ as $\operatorname{cofsum}(A)$. Graham, Hoffman and Hosoya (see [2]) showed the following.

Theorem 1 If $G$ is a strongly connected digraph with 2-connected blocks $G_{1}, G_{2}, \ldots, G_{r}$, then $\operatorname{cofsum}\left(D_{G}\right)=\prod_{i=1}^{r} \operatorname{cofsum}\left(D_{G_{i}}\right)$ and $\operatorname{det}\left(D_{G}\right)=\sum_{i=1}^{r} \operatorname{det}\left(D_{G_{i}}\right) \prod_{j \neq i} \operatorname{cofsum}\left(D_{G_{j}}\right)$.

Since all the $(n-1)$ blocks of any tree $T$ on $n$ vertices are $K_{2}$ 's, we can recover Graham and Pollak's result from Theorem 11 Yan and Yeh [5] showed a similar "tree structure independent" 
result for the problem of counting the number of signed permutations with a fixed number $k$ as the Spearman measure where distances are induced from an underlying tree $T$.

Bapat et al [1] obtained a $q$-analogue of Graham and Pollak's result and Sivasubramanian [4] obtained a $q$-analogue of Theorem 1 for the case when all the blocks of a graph are triangles. In this present work, we show a $q$-analogue of Theorem 1

\subsection{The $q$-analogue}

For a strongly connected digraph $G=(V, E)$, the $q$-analogue of its distance matrix $q D_{G}$ is obtained from its distance matrix $D_{G}$ by replacing all positive entries $i$ by $[i]_{q}=1+q+\cdots+q^{i-1}$ where $q$ is an indeterminate and $[0]_{q}=0$. Let the distance between vertices $u$ and $v$ in $G$ be denoted as $d_{u, v}$ and let the cofactor matrix (see Section 2 for definitions) of $q D_{G}$ be qCOF ${ }_{G}=$ $\left(c_{u, v}\right)$. Let the rowsum of $\mathrm{qCOF}_{G}$ corresponding to row $v$ be $\operatorname{rsum}_{v}$. Given $\mathbf{w} \in V$, consider the

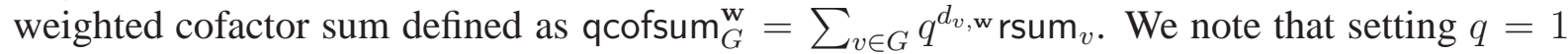
gives qcofsum $\mathbf{w}_{G}^{\mathbf{w}}=\sum_{u, v} c_{u, v}$ which is the sum of the cofactors as used in [2] and that this sum is independent of $\mathbf{w}$. In Lemma 3 , we show that qcofsum ${ }_{G}^{\mathrm{w}}$ is independent of $\mathbf{w}$ (and hence can be denoted as qcofsum ${ }_{G}$ ). In Subsection 3.1 we prove the following $q$-analogue of Graham, Hoffman and Hosoya's result.

Theorem 2 Let $G$ be a strongly connected digraph with distance matrix $D_{G}$. Let the q-analogue of $D_{G}$ be $q D_{G}$ and let $G$ have blocks $G_{1}, G_{2}, \ldots, G_{r}$. For each $1 \leqslant i \leqslant r$, let the distance matrix of $G_{i}$ and its $q$-analogue be $D_{G_{i}}$ and $q D_{G_{i}}$ respectively. Then,

1. qcofsum $_{G}=\prod_{i=1}^{r}$ qcofsum $_{G_{i}}$

2. $\operatorname{det}\left(q D_{G}\right)=\sum_{i=1}^{r} \operatorname{det}\left(q D_{G_{i}}\right) \prod_{j \neq i} \operatorname{qcofsum}_{G_{j}}$.

Thus, we show a polynomial generalisation of Graham, Hoffman and Hosoya's Theorem. We also prove a similar polynomial generalisation - when two $n \times n$ matrices $M_{1}, M_{2}$ have the same determinant, then replacing all the entries of both matrices by twice (or any scalar times) its original value clearly still gives two different matrices (say $M_{1}^{\prime}, M_{2}^{\prime}$ ) also with the same determinant value. For distance matrices, we show in Subsection 3.3 that replacing each entry by a "two-times" polynomial (and more generally by a " $k$-times" polynomial, where $k$ is a positive integer) again gives identical determinant values as polynomials.

Consider the mod-2 distance matrix of a graph, where only the parity of each entry of the distance matrix is used. We show that if two graphs $G_{1}, G_{2}$ have an identical multiset of isomorphic blocks, then the mod-2 distance matrices of $G_{1}$ and $G_{2}$ have the same determinant value, independent of the tree-like connection of their blocks. This shows that the adjacency matrix of several graphs have the same determinant value.

More generally for a positive integer $k \geqslant 3$, we first replace all the distance matrix entries by its mod- $k$ values. In the resulting matrix, if we change all entries $i$ (for $0 \leqslant i<k$ ) to $1+\zeta+\zeta^{2}+\cdots+\zeta^{i-1}$, where $\zeta$ is a primitive $k$-th root of unity, then the determinant of this (complex) matrix is again independent of the tree structure on the blocks of $G$. Subsection 3.2 contains these results. 


\section{Preliminaries}

In this section, we note a few linear algebraic preliminaries that we will need for the proof of Theorem 2 All our vectors will be column vectors and given an $n \times p$ matrix $A$, we denote its transpose by $A^{t}$. For a square matrix $A$, $\operatorname{det}(A)$ denotes its determinant.

Given an $n \times n$ matrix $A$, its row and column indices begin with 1 and we denote its $i$-th row (for $1 \leqslant i \leqslant n$ ) by $\operatorname{Row}_{i}$ and its $j$-th column (for $1 \leqslant j \leqslant n$ ) by $\operatorname{Col}_{j}$. It is convenient for determinant calculations to represent some combinations of elementary row and column operations on $A$ by multiplications of the following $n \times n$ matrices:

$$
R=\left(\begin{array}{cccc}
1 & 0 & \cdots & 0 \\
\alpha_{2} & 1 & \cdots & 0 \\
\vdots & \vdots & \ddots & \vdots \\
\alpha_{n} & 0 & \cdots & 1
\end{array}\right) \text { and } C=\left(\begin{array}{cccc}
1 & \beta_{2} & \cdots & \beta_{n} \\
0 & 1 & \cdots & 0 \\
\vdots & \vdots & \ddots & \vdots \\
0 & 0 & \cdots & 1
\end{array}\right)
$$

It follows that $R A C$ is the result of the following elementary row and column operations on $A$ performed in any order: $\operatorname{Row}_{i}:=\operatorname{Row}_{i}+\alpha_{i} \operatorname{Row}_{1}$ and $\operatorname{Col}_{i}:=\operatorname{Col}_{i}+\beta_{i} \operatorname{Col}_{1}$ for $2 \leqslant i \leqslant n$.

Given an $n \times n$ matrix $A$ and $n \times 1$ vectors $\rho$ and $\tau$, we will need to find $\operatorname{det}\left(A+x \rho \tau^{t}\right)$ where $x$ is a fresh variable, not occurring in $A, \tau$ or $\rho$. We will restrict attention to vectors $\rho, \tau$ where both $\rho_{1} \neq 0$ and $\tau_{1} \neq 0$. Let $\mathrm{cA}=\left(A_{i, j}\right)$ be the cofactor matrix of $A$ with $A_{i, j}$ for $1 \leqslant i, j \leqslant n$ denoting the cofactor at position $(i, j)$. Specifically, $A_{i, j}$ is $(-1)^{i+j}$ times the determinant of the submatrix of $A$ obtained by deleting Row $_{i}$ and $\mathrm{Col}_{j}$. Lastly, define $C_{\rho, \tau}(\mathrm{cA})=\rho^{t} \mathrm{cA} \tau$.

Lemma 1 The coefficient of $x$ in $\operatorname{det}\left(A+x \rho \tau^{t}\right)$ is $C_{\rho, \tau}(\mathrm{cA})$

Proof: The coefficient of $x$ in $\operatorname{det}\left(A+x \rho \tau^{t}\right)$ is $\sum_{i, j} \rho_{i} \tau_{j} A_{i, j}$. (This follows by observing that the only way to get an $x$ in the determinant expansion is to choose $x \rho_{i} \tau_{j}$ from the $i$-th row and $j$-th column and non- $x$ terms from other rows and columns.)

Let $\tilde{A}$ be obtained from an $n \times n$ matrix $A$ by performing $\operatorname{Row}_{i}:=\operatorname{Row}_{i}-\frac{\rho_{i}}{\rho_{1}} \operatorname{Row}_{1}$ for $2 \leqslant i \leqslant n$ and then performing $\mathrm{Col}_{i}:=\mathrm{Col}_{i}-\frac{\tau_{i}}{\tau_{1}} \mathrm{Col}_{1}$. Let

$$
R=\left(\begin{array}{cccc}
1 & 0 & \cdots & 0 \\
-\frac{\rho_{2}}{\rho_{1}} & 1 & \cdots & 0 \\
\vdots & \vdots & \ddots & \vdots \\
-\frac{\rho_{n}}{\rho_{1}} & 0 & \cdots & 1
\end{array}\right) \text { and } C=\left(\begin{array}{cccc}
1 & -\frac{\tau_{2}}{\tau_{1}} & \cdots & -\frac{\tau_{n}}{\tau_{1}} \\
0 & 1 & \cdots & 0 \\
\vdots & \vdots & \ddots & \vdots \\
0 & 0 & \cdots & 1
\end{array}\right)
$$

Celarly, $\tilde{A}=R A C$. We will use the matrices $R$ and $C$ again in this work and though they depend on the vectors $\rho$ and $\tau$, instead of using a more correct subscripted notation $R_{\rho}$ and $C_{\tau}$, we will define vectors $\rho$ and $\tau$ and only then use $R, C$. In our proof of Theorem [ we will apply this notation to cases with $A=q D_{G}$ and with $A$ being each of two principal submatrices of $q D_{G}$ with only index 1 in common; vertex 1 will be the separator between one block and the rest of the graph $G$. In each of these three cases, the vertices of the appropriate subgraph of $G$ will be labelled by the indices of $A, R, C, \mathrm{cA}, \rho$ and $\tau$ and these indices are used in the multiplications defining $C_{\rho, \tau}(\mathrm{cA})=\rho^{t} \mathrm{cA} \tau$ and $\tilde{M}=R M C$ (for $M=A$ and others). The common vertex has index 1 . In all cases, the cofactor of $\tilde{A}$ at position $(1,1)$ is denoted by $\tilde{A}_{1,1}$. 
Lemma $2 \rho_{1} \tau_{1} \widetilde{A}_{1,1}=C_{\rho, \tau}(\mathrm{cA})$

Proof: $\quad$ Since $R$ and $C$ have determinant $1, \operatorname{det}\left(A+x \rho \tau^{t}\right)=\operatorname{det}\left(R\left(A+x \rho \tau^{t}\right) C\right)=$ $\operatorname{det}(R A C+M)=\operatorname{det}(\tilde{A}+M)$, where

$$
M=\left(\begin{array}{ccc}
x \rho_{1} \tau_{1} & \cdots & 0 \\
0 & \cdots & 0 \\
\vdots & \ddots & \vdots \\
0 & \cdots & 0
\end{array}\right)
$$

Therefore, the coefficient of $x$ in $\operatorname{det}\left(A+x \rho \tau^{t}\right)$ is $\rho_{1} \tau_{1} \widetilde{A}_{1,1}$. The proof is complete by combining with Lemma11

\section{The $q$-analogue}

\subsection{Proofs of results}

With the notation of Section 1 , we begin with the Lemma below.

Lemma 3 For vertices $u_{1}, u_{2} \in G, u_{1} \neq u_{2}$, qcofsum $u_{G}^{u_{1}}=$ qcofsum $_{G}^{u_{2}}$. Thus, qcofsum $v_{G}^{v}$ is independent of the vertex $v$. Further, for all $u \in G$, $\operatorname{qcofsum}_{G}^{u}=(q-1) \operatorname{det}\left(q D_{G}\right)+\operatorname{cofsum}\left(q D_{G}\right)$, where cofsum $\left(q D_{G}\right)=\sum_{u, v} c_{u, v}$ is the sum of the cofactors of $q D_{G}$.

Proof: We recall that $q D_{G}$ is the $q$-analogue of the distance matrix $D_{G}=\left(d_{u, v}\right)$ of $G$ and $\mathrm{qCOF}_{G}=\left(c_{u, v}\right)$ is the cofactor matrix of $q D_{G}$. For two vertices $u, v \in G, d_{u, v}$ is the distance between them and $\left[d_{u, v}\right]_{q}=1+q+q^{2}+\cdots+q^{d_{u, v}-1}$. Let rsum ${ }_{v}$ be the row-sum of qCOF $G$ corresponding to row $v$ and for a vertex $u$, qcofsum ${ }_{G}^{u}=\sum_{v} q^{d_{v, u}} \operatorname{rsum}_{v}$

Elementary properties of the determinant and the adjugate imply for all vertices $u \in G$, $\operatorname{det}\left(q D_{G}\right)=\sum_{v \in G}\left[d_{v, u}\right]_{q} \cdot c_{v, u}=\sum_{v \in G}\left[d_{v, u}\right]_{q} \cdot \operatorname{rsum}_{v}$. Thus,

$$
\begin{aligned}
(q-1) \operatorname{det}\left(q D_{G}\right) & =\sum_{v \in G}(q-1)\left[d_{v, u}\right]_{q} \cdot \operatorname{rsum}_{v} \\
& =\sum_{v \in G}\left(q^{d_{v, u}}-1\right) \cdot \operatorname{rsum}_{v} \\
& =\operatorname{qcofsum}_{G}^{u}-\operatorname{cofsum}\left(q D_{G}\right)
\end{aligned}
$$

This completes the proof.

For simplicity, $d_{i, j}$ denotes $d_{v_{i}, v_{j}}$ for vertices $v_{i}, v_{j}$ in any graph and sometimes, the index $i$ will be identified with vertex $v_{i}$. Lemma 3 can be stated in the following alternate way. For a strongly connected digraph $G$, let $\mathrm{ED}_{G}=\left(e_{u, v}\right)$ be its exponential distance matrix defined as $e_{u, v}=q^{d_{u, v}}$ where $d_{u, v}$ is the distance between $u$ and $v, q$ is an indeterminate and $q^{0}=1$. 
Corollary 1 Consider the matrix $M_{G}=\mathrm{ED}_{G}^{t} \cdot \mathrm{qCOF}_{G}$. The all-ones vector $\mathbb{1}$, of dimension $|V(G)| \times 1$ is an eigenvector of $M_{G}$ corresponding to eigenvalue qcofsum ${ }_{G}$.

Proof: Let RS be the $|V(G)| \times 1$ vector with $\mathrm{RS}_{v}=$ rsum $_{v}$. Clearly, $\mathrm{qCOF}_{G} \cdot \mathbb{1}=\mathrm{RS}$ and $\left(\mathrm{ED}_{G}^{t} \cdot \mathrm{RS}\right)_{v}=\sum_{u} q^{d_{u, v}}$ rsum $_{u}=$ qcofsum $_{G}$. The proof follows.

We note the following lemma similar to the lemma in [2]. We recall the $q$-weighted cofactor

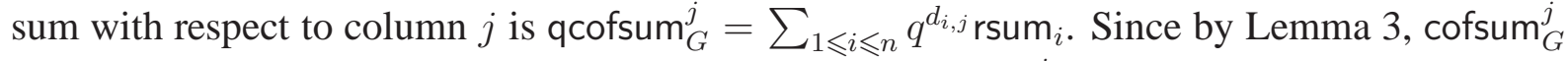
is independent of $j$, we fix $j=1$ and write $\operatorname{cofsum}_{G}=\operatorname{cofsum}_{G}^{j}$. We will use Lemma2 with

$$
A=q D_{G}, \rho^{t}=\left[1, q^{d_{2,1}}, q^{d_{3,1}}, \ldots, q^{d_{n, 1}}\right] \text { and } \tau^{t}=\mathbb{1} .
$$

These values for the $\rho_{i}$ 's and the $\tau_{i}$ 's define the matrices $R, C$ and thus $\widetilde{q D_{G}}$. It is simple to see from the definition that qcofsum ${ }_{G}=\mathrm{qcofsum}_{G}^{1}=C_{\rho, \tau}\left(\mathrm{q} \mathrm{COF}_{G}\right)$, where we recall $C_{\rho, \tau}\left(\mathrm{q} \mathrm{COF}_{G}\right)=\rho^{t}\left(\mathrm{qCOF}_{G}\right) \tau$. The following lemma gives the cofactor of $q \tilde{D}_{G}$ at position $(1,1)$.

Lemma 4 With the above notation, $C_{\rho, \tau}\left(\mathrm{q} \mathrm{COF}_{G}\right)=\widetilde{\left(q D_{G}\right)_{1,1}}$.

Proof: Follows from Lemma 2 by noting $\rho_{1}=\tau_{1}=1$.

Proof: (Of Theorem 2) Pairs of distinct blocks have at most one vertex in common; the common vertex joining two adjacent blocks is called a cut-vertex. Among the blocks of $G$, let $H$ be a block which has only one cut-vertex. We call such blocks as leaf-blocks. Clearly, leaf-blocks exist and let $H$ be a leaf block connected to the rest of $G$ along a cut-vertex. Let us label the vertices so that this cut-vertex is labelled by 1 , so when $v_{i}$ denotes a vertex of $H$ and $u_{j}$ denotes a vertex of $G^{\prime}, v_{1}=u_{1}=1$ denotes this cut-vertex in $G$. We recall the cofactor matrix $\mathrm{qCOF}_{H}=\left(c_{u, v}^{H}\right)$ of $q D_{H}$, and the $q$-weighted cofactor sum qcofsum ${ }_{H}$ defined above.

Let $|H|=k$ and $V(H)=\left\{1, v_{2}, \ldots, v_{k}\right\}$. We recall $G^{\prime}=G-(H-\{1\})$, and if $\left|G^{\prime}\right|=r$, let $V\left(G^{\prime}\right)=\left\{1, u_{2}, \ldots, u_{r}\right\}$. Let us introduce the following notation. Row vector $\overline{[a]_{q}}=$ $\left(\left[a_{2}\right]_{q}, \ldots,\left[a_{k}\right]_{q}\right)$, row vector $\overline{[f]_{q}}=\left(\left[f_{2}\right]_{q}, \ldots,\left[f_{r}\right]_{q}\right)$, column vector $\overline{[b]_{q}}=\left(\left[b_{2}\right]_{q}, \ldots,\left[b_{k}\right]_{q}\right)^{t}$ and column vector $\overline{[g]_{q}}=\left(\left[g_{2}\right]_{q}, \ldots,\left[g_{r}\right]_{q}\right)^{t}$. We also use $(M(i, j))$ to denote the matrix with entries $M(i, j)$ and various ranges of indices. We now verify that given the following block decompositions

$$
q D_{H}=\left(\begin{array}{cc}
0 & \overline{[a]_{q}} \\
{[b]_{q}} & P
\end{array}\right) \text { and } q D_{G^{\prime}}=\left(\begin{array}{cc}
0 & \overline{[f]_{q}} \\
{[g]_{q}} & Q
\end{array}\right)
$$

we can express

$$
q D(G)=\left(\begin{array}{c|c|c}
0 & \overline{[a]_{q}} & \overline{[f]_{q}} \\
\hline \overline{[b]_{q}} & P & \left(\left[b_{i}\right]_{q}+q^{b_{i}}\left[f_{j}\right]_{q}\right) \\
\hline \overline{[g]_{q}} & \left(\left[g_{i}\right]_{q}+q^{g_{i}}\left[a_{j}\right]_{q}\right) & Q
\end{array}\right)
$$

We must verify that $\left[d_{i, j}\right]_{q}=\left[b_{i}\right]_{q}+q^{b_{i}}\left[f_{j}\right]_{q}$ when $v_{i}, i \neq 1$ is a vertex of $H$ and $v_{j}, j \neq 1$ is a vertex of $G^{\prime}$. Consider such a pair of vertices. Since $v_{1}$ is a cut-vertex separating $H$ and $G^{\prime}$, 
the distances satisfy $d_{i, j}=d_{i, 1}+d_{1, j}$ It follows from the fact that $[n+m]_{q}=[n]_{q}+q^{n}[m]_{q}$ that $\left[d_{i, j}\right]_{q}=\left[d_{i, 1}\right]_{q}+q^{d_{i, 1}}\left[d_{1, j}\right]_{q}$. However, by the block decomposition of $q D_{H},\left[d_{i, 1}\right]_{q}=\left[b_{i}\right]_{q}$; and by the block decomposition of $q D_{G^{\prime}},\left[d_{1, j}\right]_{q}=\left[f_{j}\right]_{q}$. We verify in the same manner that $\left[d_{i, j}\right]_{q}=\left[d_{i, 1}\right]_{q}+q^{g_{i}}\left[a_{j}\right]_{q}$ when $i \neq 1$ labels a vertex of $G^{\prime}$ and $j \neq 1$ labels a vertex of $H$.

As operation $\sim$ preserves determinant, and by definition of $\widetilde{\left(q D_{G^{\prime}}\right)_{1,1}}$ and $\widetilde{\left(q D_{H}\right)_{1,1}}$, we have

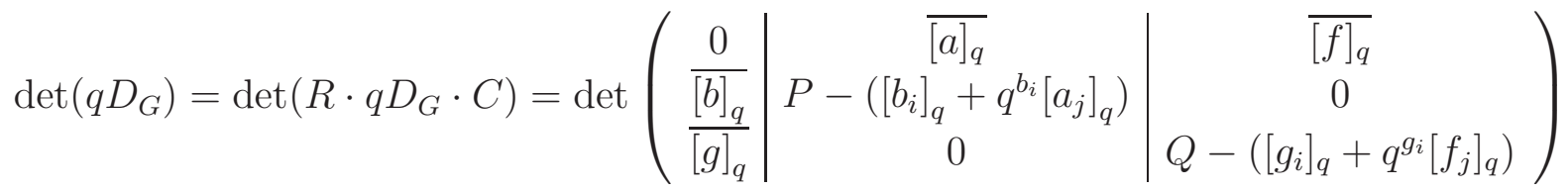

$$
\begin{aligned}
& =\operatorname{det}\left(\frac{0}{\overline{[b]_{q}}} \mid \begin{array}{c}
\overline{[a]_{q}} \\
P-\left(\left[b_{i}\right]_{q}+q^{b_{i}}\left[a_{j}\right]_{q}\right)
\end{array}\right) \cdot \operatorname{det}\left(Q-\left(\left[g_{i}\right]_{q}+q^{g_{i}}\left[f_{j}\right]_{q}\right)\right) \\
& +\operatorname{det}\left(\frac{0}{\overline{[g]_{q}}} \mid \begin{array}{c}
\overline{[f]_{q}} \\
Q-\left(\left[g_{i}\right]_{q}+q^{g_{i}}\left[f_{j}\right]_{q}\right)
\end{array}\right) \cdot \operatorname{det}\left(P-\left(\left[b_{i}\right]_{q}+q^{b_{i}}\left[a_{j}\right]_{q}\right)\right) \\
& =\operatorname{det}\left(\widetilde{q D_{H}}\right) \cdot \widetilde{\left(q D_{G^{\prime}}\right)_{1,1}}+\operatorname{det}\left(\widetilde{q D_{G^{\prime}}}\right) \cdot \widetilde{\left(q D_{H}\right)_{1,1}} \\
& =\operatorname{det}\left(q D_{H}\right) \cdot \operatorname{qcofsum}_{q D_{G^{\prime}}}+\operatorname{det}\left(q D_{G^{\prime}}\right) \cdot \operatorname{qcofsum}_{q D_{H}}
\end{aligned}
$$

where the last line follows from Lemma4 with the observation that $\rho, \tau$ restricted to the vertices of $H, G^{\prime}$ are as in Equation 1 with the dimensions of the restrictions of $\rho, \tau$ matching that of either $A=q D_{H}$ or $A=q D_{G^{\prime}}$. Using Lemma 4 again, we note that

$$
\begin{aligned}
\operatorname{qcofsum}_{q D_{G}} & =\operatorname{det}\left(\begin{array}{c}
P-\left(\left[b_{i}\right]_{q}+q^{b_{i}}\left[a_{j}\right]_{q}\right) \\
0
\end{array} \mid \begin{array}{c}
0 \\
\\
=
\end{array} \begin{array}{c}
\operatorname{det}\left(P-\left(\left[g_{i}\right]_{q}+q^{g_{i}}\left[f_{j}\right]_{q}\right)\right.
\end{array}\right) \\
& \left.\left.=\widetilde{\left(q b_{i}\right]_{q}+q^{b_{i}}}\left[a_{j, 1}\right]_{q}\right)\right) \cdot \operatorname{det}\left(Q-\left(\left[g_{i}\right]_{q}+q^{g_{i}}\left[f_{j}\right]_{q}\right)\right) \\
& =\text { qcofsum }_{q D_{H}} \cdot \text { qcofsum }_{q D_{G^{\prime}}}
\end{aligned}
$$

The proof is complete.

We apply Theorem 2 to obtain a few known corollaries and some new ones as well. When $G=T$ is a tree, each block $G_{i}$ is an edge (i.e. a $K_{2}$ ). It is simple to note that qcofsum $G_{i}=$ $-(1+q)$ and $\operatorname{det}\left(D_{G_{i}}\right)=-1$. Thus, we get a $q$-analogue of Graham, Hoffman and Hosoya's result first observed by Bapat et. al [1] Corollary 5.2].

Corollary 2 (Corollary 5.2, [1]) When $G$ is a tree on $n$ vertices, then $\operatorname{det}\left(q D_{G}\right)=(-1)^{n-1}(n-$ 1) $(1+q)^{n-2}$.

When each block of $G$, is a 3 -clique(i.e. a $K_{3}$ ), we get

$$
D_{G_{i}}=\left(\begin{array}{ccc}
0 & 1 & 1 \\
1 & 0 & 1 \\
1 & 1 & 0
\end{array}\right)
$$


thus qcofsum $G_{i}=(1+2 q)$ and $\operatorname{det}\left(D_{G_{i}}\right)=2$. From this, we recover the following result of Sivasubramanian [4]. More generally, when each block of $G$ is an $r$-clique (ie $K_{r}$ ), then $D_{G_{i}}=$ $J-I$, where $J$ is the matrix of all ones and $I$ is the identity matrix, both of dimension $r \times r$. It is simple to check that qcofsum $G_{i}=(-1)^{r-1}[1+(r-1) q]$ and $\operatorname{det}\left(D_{G_{i}}\right)=(-1)^{r-1}(r-1)$.

Corollary 3 Let $G$ have $k$ blocks all of which are r-cliques (thus, G has $n=(r-1) k+1$ vertices).

- When $r=3, \operatorname{det}\left(q D_{G}\right)=2 k(1+2 q)^{k-1}$. (4 Corollary 3].)

- More generally for any $r$, $\operatorname{det}\left(q D_{G}\right)=(-1)^{n-1}[(r-1) \cdot k][1+(r-1) q]^{k-1}$.

\subsection{Mod $k$ distances, setting values to $q$}

In this subsection, by setting values to $q$, we get a few pleasing corollaries about some modifications of the distance matrix of graphs, some of which seem non obvious.

If we set $q=-1$, then it is easy to check that for odd $i,[i]_{q}=1$ and for even $i,[i]_{q}=0$. Let $G$ be a connected graph with distance matrix $D_{G}$ and let $q D_{G}$ be the $q$-analogue of $D_{G}$. If we set $q=-1$ in all entries of $q D_{G}$, this operation corresponds to considering the distance matrix $D_{G}$ with all entries modulo 2 .

Theorem 3 Let $G$ and $H$ be graphs with an identical multiset of isomorphic blocks (they may differ in the tree structure of the connection among these blocks). Let $D_{G}^{\prime}$ and $D_{H}^{\prime}$ be the mod-2 distance matrices (where all distances are all considered modulo 2) of $G$ and $H$ respectively. Then $\operatorname{det}\left(D_{G}^{\prime}\right)=\operatorname{det}\left(D_{H}^{\prime}\right)$.

Proof: Follows from Theorem 2 by setting $q=-1$.

Corollary 4 Let $G$ be a tree and let $D_{G}^{\prime}$ be its mod-2 distance matrix where all distances are all considered modulo 2. Then $D_{G}^{\prime}$ is singular (ie $\operatorname{det}\left(D_{G}^{\prime}\right)=0$ ).

We get the following pleasant mod-2 analogue of Corollary 3 for which simple proofs would be interesting.

Corollary 5 Let $G$ be a graph with $k$ blocks, all of which are r-cliques (ie $K_{r}$ 's), and let $D_{G}^{\prime}$ be its mod-2 distance matrix (i.e. where each entry is considered modulo 2).

- If $r=3, \operatorname{det}\left(D_{G}^{\prime}\right)=2 k(-1)^{k-1}$.

- For a general $r$, det $\left(D_{G}^{\prime}\right)=(r-1) k(-r)^{n+k-2}$.

Remark 1 Theorem 3 answers the following question. Akin to determinant of the distance matrices of some graphs being equal, are there graphs such that the determinant of their adjacency matrices are identical? Since a mod-2 distance matrix has 0-1 entries, Theorem 3 gives families of graphs whose adjacency matrices have the same determinant. It would be interesting to see if there is some structure or some description of all or even a subset of the graphs which arise in this mod-2 manner from the distance matrix of graphs having an identical multiset of isomorphic blocks. 
Just as we set the value $q=-1$, we set other values to $q$ and get further corollaries. The following corollary was suggested by the referee. For a positive integer $k$, let $\zeta$ be a primitive $k$-th root of unity. Clearly setting $q=\zeta$ corresponds to the following operation: replace each positive entry $i$ in the distance matrix of $G$ by $1+\zeta+\cdots+\zeta^{(i \bmod k)-1}$. Setting $q=-1$ corresponds to this operation with $k=2$. Thus, we get the following.

Corollary 6 Let $G$ and $H$ be graphs with an identical multiset of isomorphic blocks (they may differ in the tree structure of the connection among these blocks). For any fixed positive integer $k$, let $\zeta$ be a primitive $k$-th root of unity. Let $D_{G}^{\prime}$ and $D_{H}^{\prime}$ be the mod- $k$ distance matrices of $G$ and $H$ respectively, where all positive distances $i$ are replaced by $1+\zeta+\cdots+\zeta^{i-1}$. Then $\operatorname{det}\left(D_{G}^{\prime}\right)=\operatorname{det}\left(D_{H}^{\prime}\right)$.

\section{3 $[k d]_{q}$-analogues}

In this subsection, for any positive integer $k$, we consider $k D_{q}$ analogues of $D$, where we replace positive integers $i$ in $D$ by $[k i]_{q}=1+q+q^{2}+\cdots+q^{k i-1}$. Thus, we replace all entries $[i]_{q}$ in $q D_{G}$ by $[k i]_{q}$ to get $k D_{q}$. It is easy to see that $[k i]_{q}=\left(1+q^{i}+q^{2 i}+\cdots+q^{(k-1) i}\right)[i]_{q}$. Thus, if we define $[k]_{q^{i}}$ analogously as $1+q^{i}+q^{2 i}+\cdots+q^{(k-1) i}$, we get $[k i]_{q}=[k]_{q^{i}}[i]_{q}$. It can be checked that with weights $q^{k \cdot d_{u, v}}$ multiplying $\operatorname{rsum}_{v}$, we get qcofsum ${ }_{k G}^{u}$, independent of vertex $u$. The proofs of all Lemmata and Theorem 2 in Subsection 3.1 go through as before. We omit the details and state the following result for trees in the case $k=2$.

Corollary 7 Let $T$ be a tree on $n$ vertices and let $D$ be its distance matrix. Let $2 D_{q}$ be the polynomial matrix obtained from $D$ by replacing all entries $i$ by $[2 i]_{q}=1+q+q^{2}+\cdots+q^{2 i-1}$. Then, $\operatorname{det}\left(2 D_{q}\right)=(-1)^{n-1}(n-1)(1+q)^{n}\left(1+q^{2}\right)^{n-2}$.

Proof: Follows by observing that for $H=K_{2}$, $\operatorname{det}\left(2 H_{q}\right)=-(1+q)^{2}$ and that qcofsum ${ }_{2 H_{q}}=$ $-\left(1+q^{2}\right)(1+q)$

We end with a question. Just as multiplying all entries of an $n \times n$ matrix by a factor $\alpha$ results in multiplication of its determinant by $\alpha^{n}$, multiplying just the elements of a subset $S$ with $|S|=k$ of the rows by $\alpha$ results in multiplication of its determinant by $\alpha^{k}$. It would be interesting to see if for some distinct trees $T_{1}, T_{2}$, some subsets $S_{1}, S_{2}$ with $\left|S_{1}\right|=\left|S_{2}\right|$ exist such that the $q$-analogue of just the rows of $S_{i}$ in $T_{i}$ can be multiplied to get identical polynomials for the determinant of the distance matrix.

\section{Acknowledgement}

Some Theorems in this work were in their conjecture form, tested using the computer package "Sage". We thank the authors for generously releasing Sage as an open-source package. The url http://www.math.iitb.ac.in/ krishnan/q_analog_GHH/ has the Sage worksheets containing computations which led to the conjectures and results in this work.

We sincerely thank the anonymous referee for detailed, insightful comments and for pointing out several inaccuracies which has markedly improved the presentation. Further, the referee pointed out Corollary 6 and suggested giving a link to the Sage worksheets. 


\section{References}

[1] BApAt, R. B., LAL, A. K., And PATI, S. A $q$-analog of the distance matrix of a tree. Linear Algebra and its Applications 416 (2006), 799-814.

[2] Graham, R. L., Hoffman, A. J., And Hosoya, H. On the distance matrix of a directed graph. Journal of Graph Theory 1 (1977), 85-88.

[3] Graham, R. L., And Pollak, H. O. On the addressing problem for loop switching. Bell System Tech. J 50 (1971), 2495-2519.

[4] Sivasubramanian, S. q-analogs of distance matrices of hypertrees. Linear Algebra and Applications 431(8) (2009), 1234-1248.

[5] YAN, W., AND YEH, Y.-N. The determinants of $q$-distance matrices of trees and two quantities relating to permutations. Advances in Applied Math. 39 (2007), 311-321. 